\title{
EVALUATING DIMENSION ACCURACY OF THREE DIFFERENTLY FABRICATED DENTURE BASE MATERIALS
}

\author{
Ahmed Mohamed El-Sayed*, Amr Salah El Din ${ }^{* *}$ and Inass Abdel Rahman AbuElmagd ${ }^{* * *}$
}

\begin{abstract}
Objective: to compare the accuracy of three different denture base materials used in maxillary edentulous arch

Materials and Methods: ten patients were selected, with completely edentulous arches, for each patient three finial PMMA denture bases were done using different construction technique pack and press, computer aided design/ computer aided manufacturing CAD/CAM milled and selective laser sintering (SLS) PMMA denture base All denture bases in this study were submitted for CBCT scanning for dimension accuracy and volume linear evaluation.
\end{abstract}

Results: The mean and standard deviation values were calculated for each group in each test. Data were explored for normality using Kolmogorov-Smirnov and Shapiro-Wilk tests, data showed parametric (normal) distribution. A statistically significant difference was found between (Heat cure acrylic denture), (CAD/CAM denture) and (Laser sintering denture)

Conclusion: also press and pack technique is more time consuming regarding number of visits but less dimensional changes than CAD/CAM and (SLS) selective laser sintering.

KEY WORDS: denture base, dimension accuracy, CAD/CAM, SLS\& dimension accuracy

\section{INTRODUCTION}

Computer-aided design and computer-aided manufacturing $(\mathrm{CAD} / \mathrm{CAM})$ is considered as a new technique for denture fabrication, by the use of prepolymerized blocks of polymethyl methacrylate (PMMA), by the using of CAD/CAM Computer software and 5-axis milling. Which have become widely used now days. ${ }^{(1)}$
CAD/CAM dentures have many benefits for both the clinician and the patient, as the dentures normally needed 5 visits involving many hours of the dentist's, technician's, and patient's time. While CAD/CAM dentures can be completed in as few as 2 visits, saving much time. In addition that the Patient records are digitized and Stored, so if any problem occur to the patient lose or fracture a

\footnotetext{
* Associate Professor of Removable Prosthodontics, Faculty of Dentistry, Cairo University.

** Lecturer of Removable Prosthodontics, Faculty of Dentistry, Fayoum University.

*** Associate Professor of of Oral Radiology, Faculty of Dentistry, Fayoum University.
} 
complete denture, a new and identical replacement prosthesis can be fabricated without making any new clinical records. ${ }^{(2)}$

Moreover some clinical limitations and disadvantageswere reported on $\mathrm{CAD} / \mathrm{CAM}$ complete removable dentures, such as manufacturing challenges caused by making impression, establishing occlusal vertical dimension (OVD), maxillomandibular relation (MMR) transfer, inability to define the mandibular occlusal plane, expensive materials, and increased laboratory costs in compare to conventional methods, And also if the CAD/CAM dentures are not well designed, they may show concerns, such as the short borders of denture bases and a lack of close tissue contact, which reduces retention. ${ }^{(3,4)}$

But recently, Current innovations and developments in dental technology overcome these limitations and allow the fabrication of removable dentures by using CAD/CAM from start to finish, so provide less traumatic experience for the patients for better fitting dentures. ${ }^{(5)}$

But also there, are few researches have been. published comparing the processing distortion of traditional techniques and the CAD/CAM fabrication technique. The purpose of this study was to compare the denture base adaptation of pack and press, CAD/CAM techniques of fabricating dentures to determine which process produces the most accurate and reproducible prostheses. The null hypothesis was that no differences in processing deformation would be found among the 2 techniques with regard to accuracy and reproducibility. ${ }^{(6)}$

The selective laser sintering (SLS) is an additive manufacturing technique used for the low volume production of prototype models and functional components. It was developed and patented by Dr. Carl Deckard and Dr. Joe Beaman in the mid-1980s. It involves the use of a high power laser to fuse small particles of plastic, metal, ceramic or glass powders into a mass that has a desired three dimensional Shape, as it fuses powdered material by scanning cross-sections. Generated from a 3-D digital description of the part on surface of a powder bed. After each cross-section is scanned, powder bed is lowered by one layer thickness, a new layer of material is applied on top, and the process is repeated until the part is completed SLS machine preheats. The bulk powder material in powder bed below its melting point by infrared heating in order to minimize thermal distortion (curling) and facilitate fusion to the previous layer.

Various materials can be used such as fine polymeric powder - polystyrene, polycarbonate or polyamide etc within the range of 20 to 100 micrometer diameter. The laser is modulated in such away that only those grains, which are in direct contact with the beam, are affected, and also from the advantage of (SLS) that support structures are not required as the un-sintered powder remains at the places of support structure. It is cleaned away and can be recycled once the model is complete. ${ }^{(7)}$

There are many methods used to evaluate the amount of dimensional change that occurs during denture processing. These have included 2-dimensional and 3-dimensional measurements with differing degrees of complexity. Recently, the use of laser and contact scanners has become a popular way to .measure denture base deformation. With the use of these technologies, scanned files can be superimposed and analyzed using advanced computer software. Studies have demonstrated the validity of these evaluation methods. ${ }^{(8)}$

\section{MATERIALS AND METHODS}

Ten patients were selected, with completely edentulous arches; the upper arch was examined to confirm the presence of regular bone anatomy, and firm overlying mucosa.

For each patient upper primary impressions was made from alginate impression material ${ }^{\bullet}$ in

\footnotetext{
* Cavex alginate, Cavex Holland, BV, Netherlands.
} 
properly selected and adjusted stock trays using alginate impression material, and the impression was poured in dental stone* to obtain the study casts. Self-cure acrylic resin special tray was constructed on the diagnostic casts.

Secondary impression was made in a Special tray was constructed from self cure acrylic resin, border molding was made using green stick compound ${ }^{* *}$, and the final impression was made with zinc oxide and eugenol impression material ${ }^{* * *}$. The impression was then left to set according to the manufacturer's instructions, and then the impressions were poured in type IV dental stone ${ }^{* * * * *}$, using a vacuum mixer, at a fixed room temperature, then the dental stone was left to set according to the manufacturer's instructions.

The master cast was duplicated using silicone based duplication material ${ }^{* * * * *}$, and 2 stone casts were made using a Type IV scannable dental stone ${ }^{* * * * * *}$ following the same condition of pouring master cast to facilitate comparative study and to avoid any bias during study.

For each patient three final denture bases of different colour were made, the first; red was made with conventional pack and press technique, the second; gray was made by CAD/CAM milling and the third; clear was made by selective laser sintering (SLS).

The first denture base was made on master cast while the second and third were made on the duplicated casts.

All denture bases under this study were submitted for CBCT scanning for volume and linear evaluation. CBCT images were acquired using a
Next Generation iCAT scanner (ISI, USA). A scout view was obtained and adjustments were made to ensure that the samples were correctly aligned in the scanner according to adjustment light beam before acquisition and intended field of view is obtained. The machine is supplied with Amorphous Silicon Flat Panel Sensor with Cesium Iodide (CsI) scintillator, $0.5 \mathrm{~mm}$ focal spot size, 14 Bit gray scale resolution, and operating at the following protocol for all the scans of the study:

$\begin{array}{ll}\text { Tube voltage } & 120 \mathrm{kVp} \\ \text { Milliampere } & 7 \mathrm{~mA} \\ \text { Voxel size } & 0.125 \mathrm{~mm} \\ \text { Scanning time } & 26.9 \text { seconds } \\ \text { Field of view } & 4 \mathrm{~cm} \text { Height } * 16 \mathrm{~cm} \text { Diameter }\end{array}$

After acquisition, data were exported and transferred in DICOM format and downloaded via a Compact Disk (CD) to a personal computer for analysis, where, mimics software (version 17; materialize, Belgium) was used for segmenting the scanned objects into separate volumetric elements. Definitive threshold was assigned to select certain value for all samples and its volume was automatically calculated and recorded. For linear measurement Ondemand 3D App software was utilized (Cybermed, S Korea). Fusion module was chosen to align each pair of models, and then linear measurements were taken at the same level of the two sample simultaneously. After finishing recording measurements, a new model was imported at the fusion module, and the same measurements were recorded again at the same levels. Fig. (1-5)

\footnotetext{
* Lab stone Miles dental product,Miles,inc,USA..

** Perfectin, S.A.I.C., HUBAC, BUENESAIRES, Argentina.

*** Cavex impression paste, Cavex Hollad by P.O. Box 852, 2003 RW.

**** lab stone, Miles dental product, Miles, INC, South bend,USA.

$* * * * *$ Zhermack Elite double 16 fast silicon for duplicating ,badia polesine-italy.

****** Diamond Die ${ }^{\mathrm{TM}}$ Scan Stone, Hi-Tec Dental Products.
} 


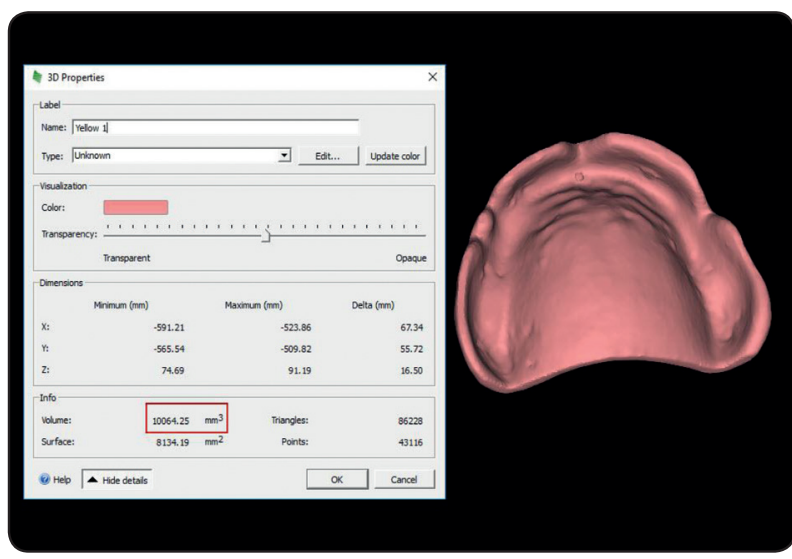

Fig (1) conventional pack and press dentur base

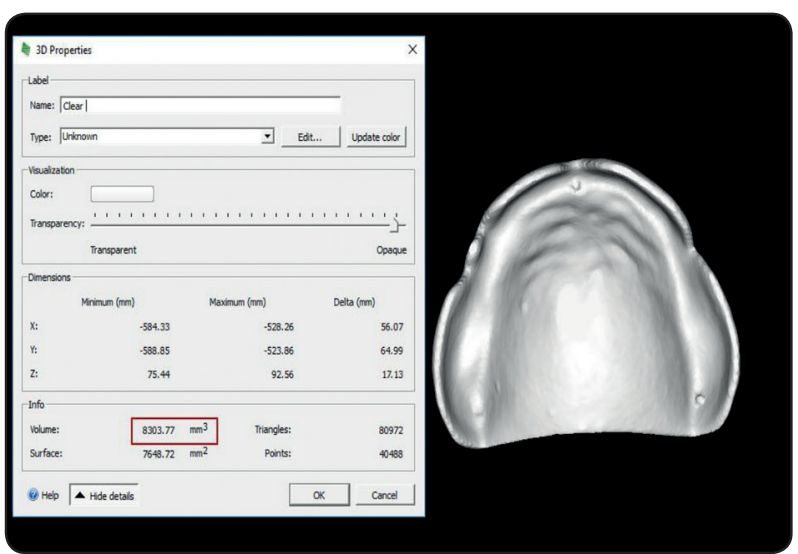

Fig (3) selective laser sintering(SLS) denture base

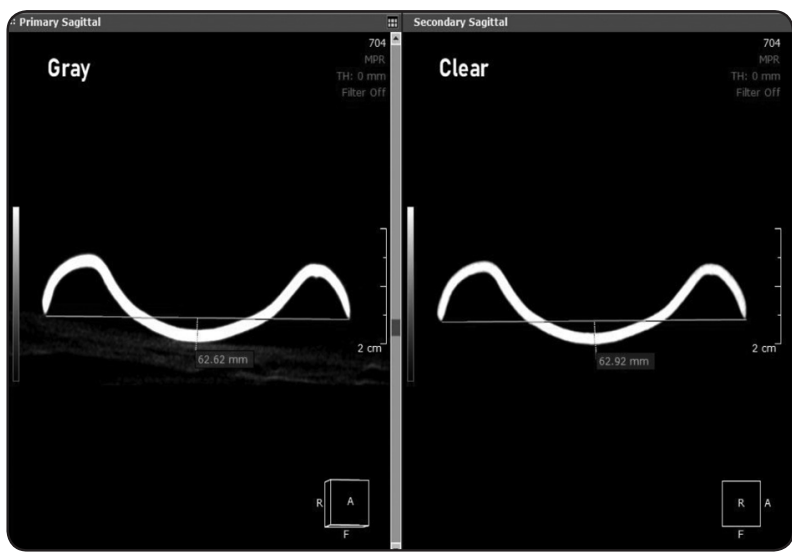

Fig. (5) linear mesurments of both CAD/CAM and SLS

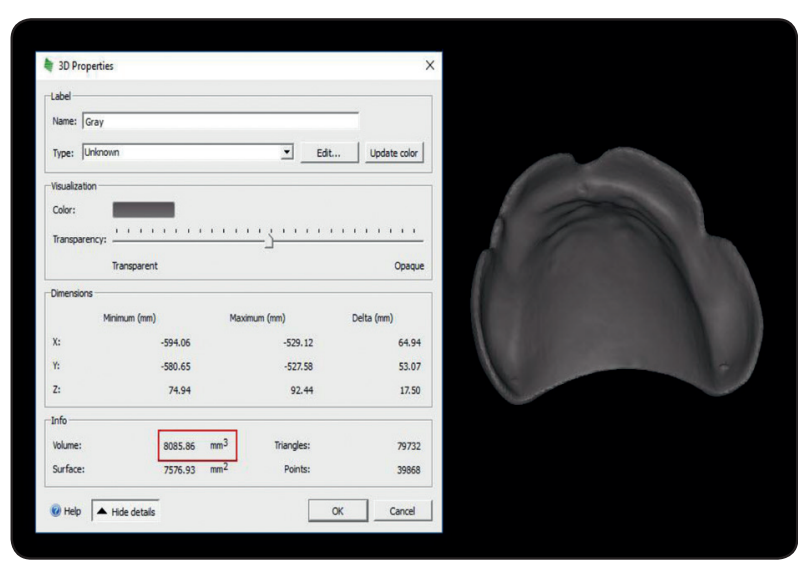

Fig. (2) CAD/CAM denture base

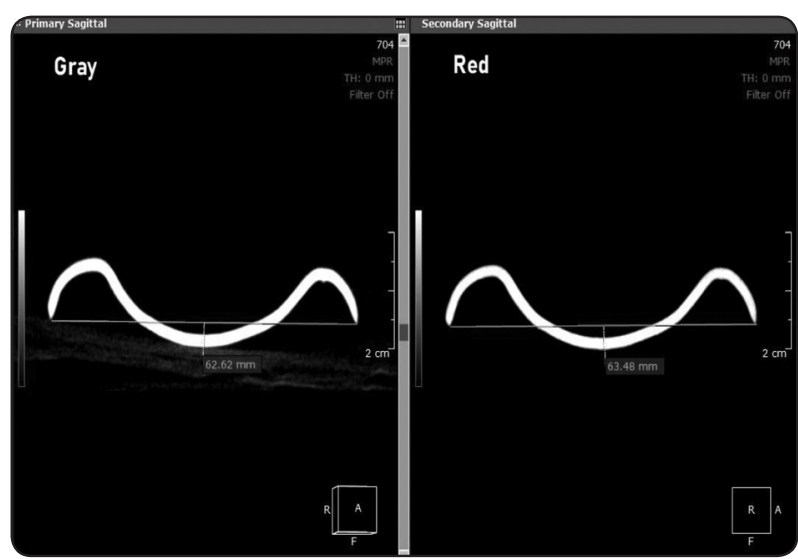

Fig (4) linear mesurments of both CAD/CAM and conventional pack and press

\section{RESULTS}

The mean and standard deviation values were calculated for each group in each test. Data were explored for normality using Kolmogorov-Smirnov and Shapiro-Wilk tests, data showed parametric (normal) distribution.

One-way ANOVA followed by Tukey post-hoc test was used to compare between more than two groups in non-related samples.

The significance level was set at $\mathrm{P} \leq 0.05$. Statistical analysis was performed with IBM® SPSS ${ }^{\circledR}$ Statistics Version 20 for Windows. 


\section{Volume results:}

A statistically significant difference was found between (Heat cure acrylic denture), (CAD/CAM denture) and (Laser sintering denture) where $(p<0.001)$.

A statistically significant difference was found between (Heat cure acrylic denture) and each of (CAD/CAM denture) and (Laser sintering denture) where $(p<0.001)$.

Also, a statistically significant difference was found between (CAD/CAM denture) and (Laser sintering denture) where $(p<0.001)$.

The highest mean value was found in (Heat cure acrylic denture) followed by (CAD/CAM denture), while the least mean value was found in (Laser sintering denture).

TABLE (1): The mean, standard deviation (SD) of volume in different groups.

\begin{tabular}{|c|c|c|}
\hline \multirow{2}{*}{ Variables } & \multicolumn{2}{|c|}{ Volume } \\
\cline { 2 - 3 } & Mean & SD \\
\hline Heat cure acrylic denture & 10064.99 & 3.25 \\
\hline CAD/CAM denture & 8304.18 & 5.34 \\
\hline Laser Sintering denture & 8085.39 & 2.12 \\
\hline p-value & \multicolumn{2}{|c|}{$<0.001 *$} \\
\hline
\end{tabular}

*; significant $(p<0.05) n s ;$ non-significant $(p>0.05)$

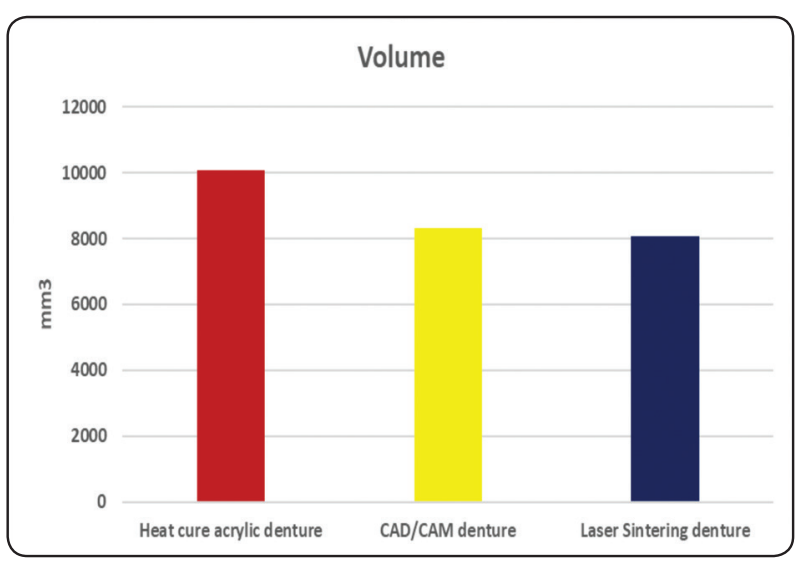

Fig. (6): Bar chart representing volume for different groups

\section{DISCUSSION}

In previously published articles comparing pack and press with $\mathrm{CAD} / \mathrm{CAM}$ in terms of volumetric changes, stated that. CAD/CAM had the narrowest dimensional distortion, which was located closest to zero and therefore was the most reproducible technique. Followed by Pack and press which had the larger range. ${ }^{(10,11)}$

In this study CBCT scanning for volume and linear evaluation was used. The CBCT images were acquired .using a Next Generation iCAT scanner so the denture bases were adapted easily and as accurately as possible.

It is clear that previous studies that evaluated the adaptation of denture bases relied on physical .measurements. But In this study, volume and linear evaluation were measure using the CBCT images.

One limitation to any study .evaluating denture adaptation in vitro is how to correlate the results in vivo. No experimental protocol exists that accurately reproduces soft tissue compression measurement. In vitro and it"s similarity in vivo. In this study, the technique that consistently provides the accurate dimension of the denture bases, which can be easly compared to each other,as theses denture bases were constructed on a master cast that is a representation of the patient's anatomy based on the clinician's impression making ability.

In this study With regard to accuracy, Pack and press is better than the CAD/CAM and SLS.

So it was suggested that pack and press is viable processing techniques for fabricating dentures than CAD/CAM and SLS. However, the use of pack and press technique takes more visits and also can result in undesirable features such as prosthetic tooth shift during processing, air entrapment, and poor bonding between the denture base and teeth. ${ }^{(12)}$ 


\section{CONCLUSIONS}

- Also press and pack technique is more time consuming regarding number of visits but less dimensional changes than $\mathrm{CAD} / \mathrm{CAM}$ and (SLS) selective laser sintering

- Farther investigation in vivo study to co-relate the accuracy of CAD/CAM , (SLS) selective laser sintering and press and pack technique to result obtained from in vitro study.

\section{REFRANCES}

1. Goodacre CJ, Garbacea A, Naylor WP, Daher T, Marchack CB, Lowry J. CAD/CAM fabricated complete denture concepts and clinical methods ofobtaining required morphological data. J Prosthet Dent, vol. 107, 2012.

2. Kattadiyil MT, Goodacre CJ, Baba NZ. CAD/CAM complete dentures: a review of two commercial fabrication systems. J Calif Dent Assoc, vol. 4 , 2013.

3. M. Bilgin, E. Baytaroglu, A. Erdem, and E. Dilber, “A review of computer-aided design/computer-aided manufacture techniques for removable denture fabrication", European Journal of Dentistry, vol. 10, 2016.

4. L. Infante, B. Yilmaz,E.McGlumphy, and I. Finge "Fabricating complete dentures with CAD/CAM technology," Journal of Prosthetic Dentistry, vol. 111, 2014.
5. M. T. Kattadiyil, R. Jekki, C. J. Goodacre, and N. Z. Baba, "Comparison of treatment outcomes in digital and conventional complete removable dental prosthesis fabrications in a predoctoral setting," Journal of Prosthetic Dentistry, vol. $114,2015$.

6. Fenlon MR, Juszczyk AS, Rodriguez JM, Curtis RV. Dimensional stability of complete denture permanent acrylic denture bases; a comparison of dimensions before and after a

7. Pandey, P.M., Pham, D.T. and Dimov, S.S. "Rapid Prototyping Technologies, Applications and Part Deposition Planning”. International Journal of Life Sciences Vol. 3, 2014.

8. Artopoulos A, Juszczyk AS, Rodriquez JM, Clark RK, Radford DR. Threedimensional processing deformation of three denture base materials. J Prosthet Dent, vol.110, 2013.

9. Second curing cycle. Eur J Prosthodont Restor Dent, vol.18, 2010 .

10. M. T. Kattadiyil, C. J. Goodacre, and N. Z. Baba, CAD/ CAM complete dentures: a review of two commercial fabrication systems, Journal of the California Dental Association, vol. 41,2013.

11. S. B. M. Patzelt, S. Vonau, S. Stampf, andW. Att, “Assessing the feasibility and accuracy of digitizing edentulous jaws," Journal of the American Dental Association, vol. 144, 2013.

12. Anusavice KJ, Shen C, Rawls HR. Phillips' science of dental materials. $12^{\text {th }}$ ed. St. Louis: Elsevier; 2013. 